\title{
Mitigating Channel Estimation Error via Cooperative Communications
}

\author{
Ahmed S. Ibrahim and K. J. Ray Liu \\ Department of Electrical and Computer Engineering, University of Maryland, College Park, MD 20742, USA.
}

\begin{abstract}
Channel estimation error problem is among the main causes of performance degradation in wireless networks. In this paper, we investigate the impact of cooperative communications on mitigating the effect of channel estimation error. Two main performance criteria, namely, the traditional outage probability and the proposed signal-to-noise ratio (SNR) gap ratio, are utilized to characterize such impact. The SNR gap ratio measures the reduction in the SNR due to channel estimation error. Taking into consideration the channel estimation error, we show that the outage probability is reduced by utilizing cooperative transmission. We also show that cooperative transmission results in lower SNR gap ratio compared to that of the direct transmission. Thus, cooperative transmission is less susceptible to the effect of channel estimation error compared to direct transmission. Finally, we illustrate that increasing the number of cooperating relays reduces the effect of the channel estimation error more.
\end{abstract}

\section{INTRODUCTION}

Recently, cooperative communications for wireless networks have gained much interest due to its ability to mitigate fading in wireless networks through achieving spatial diversity, while resolving the difficulties of installing multiple antennas on small communication terminals. Cooperative communication protocols make use of the broadcast nature of wireless channels, where a number of relay nodes are assigned to help a source in forwarding its information to its destination, hence forming a virtual antenna array. Various cooperative diversity protocols were proposed and analyzed in [1], [2], [3], [4], [5], [6], [7], [8].

In [2], Laneman et al. described various techniques of cooperative communication such as decode-and-forward and amplify-and-forward. The symbol error rate (SER) for singleand multi-node decode-and-forward cooperative technique was analyzed in [3], [4]. It was shown that the conventional cooperative communication scenario, in which each of the available $N$ relays forwards the source's information over an orthogonal channel (e.g. time or frequency slot), achieves full diversity order equal to $N+1$. However, the bandwidth efficiency drops to $1 /(N+1)$ symbols per channel use (SPCU). In [5], [6] various relay selection cooperative schemes, in which only one relay forwards the source's information, have been proposed. It was illustrated that relay-selection cooperative schemes achieve high bandwidth efficiency while guaranteeing full diversity order. Distributed space-time cooperative schemes, in which the helping relays simultaneously forward modified version of the source's information, have been proposed and analyzed in [7], [8]. It was shown that distributed cooperative schemes, under certain conditions, can achieve full diversity order.

Channel estimation error, caused possibly by Doppler shift or noise on the pilot signals, can cause dramatic performance degradation in wireless networks. In [9], it was examined that channel estimation error results in lower average signal-tonoise ratio (SNR) and higher average error rate in orthogonal frequency division multiplexing (OFDM)-based systems. In [10], a superposition coding scheme was proposed to reduce the channel estimation effect when the users have largely different SNR.

Motivated by the bad impact of channel estimation error on the direct transmission scenario, we investigate in this paper the ability of the cooperative transmission to mitigate such impact. We consider two main performance criteria to characterize the effect of cooperative communication on channel estimation error, namely, the traditional outage probability and the proposed SNR gap ratio. The SNR gap ratio quantifies the reduction in the SNR due to channel estimation error. First, we show that the outage probability is reduced due to utilizing cooperative communications in the presence of channel estimation error. Second, we illustrate that cooperative transmission reduces the SNR gap ratio compared to that of the direct transmission. We find that cooperative communication is less susceptible to channel estimation error by achieving spatial diversity via relays and distributing the total transmission power across multiple transmission phases. Moreover, increasing the number of relays reduces the effect of the channel estimation error more.

The rest of this paper is organized as follows. In the next section, we describe the system model of the communication system, taking into consideration the channel estimation error effect, and explain the problem formulation. We Study the impact of various communication scenarios on the channel estimation error in Section III. In Section IV we show some numerical results, and finally Section V concludes the paper.

\section{System Model and PRoblem Formulation}

Communication scenarios, which are based on training sequences (pilots) for channel estimation, are implemented in two consecutive phases, namely, training phase and data transmission phase. In the training phase, the channel is estimated using a known training sequence with a particular pilot power, denoted by $P_{\text {pilot }}$. A particular pilot transmission power $P_{\text {pilot }}$ results in a certain level of channel estimation error variance, referred to as $\alpha$, which is inversely proportional to the pilot transmission power. In the end of the training phase, the receiver has an estimate of the channel to be utilized in the coherent detection of the transmitted data in the following data transmission phase. In the data transmission phase, the channel estimate is fixed and does not depend on the data transmission power, $P$. Hence, the channel estimation error does not depend on the data transmission power, $P$.

The communication system under consideration is shown in Fig. 1. It consists of the source, $s$, the destination, $d$, and 


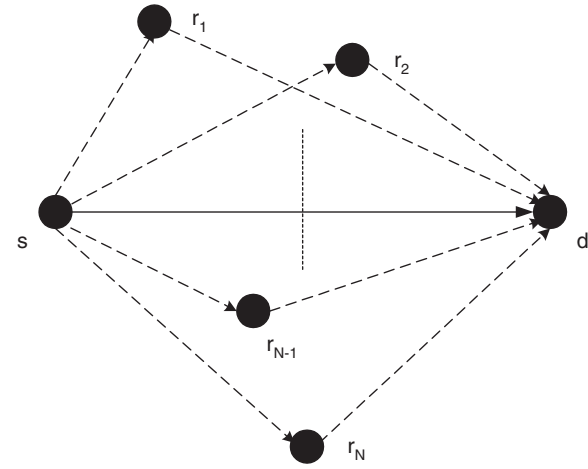

Fig. 1. Cooperative communication system with a set of $N$ relays. Solid line represents the direct transmission and dashed lines represent the cooperative transmissions via the relays.

a set of $N$ relays, $r_{1}, r_{2}, \cdots, r_{N}$. We assume that there is a fixed channel estimation error variance, $\alpha$, resulting from the training phase due to utilizing a certain level of pilot transmission power. We take into consideration the channel estimation error in the data transmission phase as follows. In the direct transmission scenario, the source sends its data symbol to the destination in one phase, which can be a time or frequency slot. The received symbol at the destination can be modeled as

$$
y_{s, d}^{D}=\sqrt{P}\left(h_{s, d}+h_{\alpha}\right) x+\sqrt{N_{0}} \eta_{\alpha},
$$

where the superscript $D$ denotes the direct transmission scenario, $x$ is the transmitted symbol with unit average energy, i.e., $|x|^{2}=1, h_{s, d}$ is the estimated source-destination channel coefficient, $h_{\alpha}$ denotes the channel estimation error, $\eta_{\alpha}$ is a zero-mean additive white Gaussian noise (AWGN) with unit variance, and $N_{0}$ is the noise variance. In [10], the additional term resulting from channel estimation error, namely $\sqrt{P} x h_{\alpha}$ in (1), was called self-noise because it represents an added noise term that scales with the data transmission power.

The channel estimation error is a summation of large number of small quantities representing the inter-carrier interference and noise, and hence it can be modeled as a Gaussian random variable via the central limit theorem [11]. Similar to [9], the channel estimation error $h_{\alpha}$ is modeled as a zero-mean complex Gaussian random variable with variance $\alpha$. Since we are to study the impact of the channel transmission error on the system performance, by fixing the transmitted symbol $x$, the additional self-noise term $\left(\sqrt{P} x h_{\alpha}\right)$ is a zero-mean complex Gaussian random variable with variance $\alpha P$. Thus, (1) can be rewritten as

$$
y_{s, d}^{D}=\sqrt{P} h_{s, d} x+\sqrt{\alpha P+N_{0}} \eta_{s, d},
$$

where $\eta_{s, d}$ is a zero-mean Gaussian random variable with unit variance. We note that the system model in (2) is similar to the one that was considered to represent the channel estimation error effect in [10].

In the $N$-relay cooperative transmission scenario, a transmission of one symbol is implemented in $N+1$ phases. In the first phase, the source broadcasts its symbol to the relays and the destination with a transmission power of $P_{0}$. Taking into consideration the channel estimation error as in (2), the received symbols at the destination and the $i$-th relay can be modeled as

$$
\begin{gathered}
y_{s, d}^{C}=\sqrt{P_{0}} h_{s, d} x+\sqrt{\alpha P_{0}+N_{0}} \eta_{s, d}, \\
y_{s, r_{i}}^{C}=\sqrt{P_{0}} h_{s, r_{i}} x+\sqrt{\alpha P_{0}+N_{0}} \eta_{s, r_{i}},
\end{gathered}
$$

respectively, where the superscript $C$ denotes the cooperative transmission scenario, $h_{s, r_{i}}$ is the estimated channel coefficient between the source and the $i$-th relay, and $\eta_{s, r_{i}}$ is a zero-mean AWGN with unit variance.

In this paper, without loss of generality we consider the decode-and-forward cooperative protocol [3], [4]. However, the system model and the following performance analysis can be easily extended to other cooperative protocols such as amplify-and-forward [2], [3]. In the decode-and-forward protocol, each relay decides whether to forward the received information or not according to the quality of the received signal. We assume that every relay can tell whether the received information is correctly decoded or not [3], [4]. If the $i$-th relay correctly decodes the received symbol, then it forwards the decoded symbol to the destination in the $(i+1)$ th phase, otherwise it remains idle. The received symbol at the destination in the $(i+1)$-th phase is given by

$$
y_{r_{i}, d}^{C}=\sqrt{\tilde{P}_{i}} h_{r_{i}, d} x+\sqrt{\alpha \tilde{P}_{i}+N_{0}} \eta_{r_{i}, d},
$$

where $\tilde{P}_{i}=P_{i}$ if the relay decodes the symbol correctly, otherwise $\tilde{P}_{i}=0, h_{r_{i}, d}$ is the estimated channel coefficient between the $i$-th relay and destination, and $\eta_{r_{i}, d}$ is a zero-mean AWGN with unit variance. The transmission powers, $P_{i}, i=$ $0,1, \cdots, N$, are allocated subject to a total power constraint of $P_{0}+\sum_{i=1}^{N} P_{i}=P$ [4]. This power constraint is imposed to guarantee a fair comparison with the direct transmission scenario.

Flat Rayleigh fading channels are considered. Let $h_{u, v}$ be a generic channel coefficient representing the channel between any two nodes, where $h_{u, v}$ is modeled as zero-mean complex Gaussian random variable with variance $\delta_{u, v}^{2}$. The channel gain squared $\left|h_{u, v}\right|^{2}$ follows an exponential random variable with mean $\delta_{u, v}^{2}$ [11]. We assume that the channel coefficients between each two nodes are independent of each other [3], [4], which can be practically achieved by deploying the nodes far enough from each other.

Below, we illustrate the performance degradation due to channel estimation error in the direct transmission case. For the direct transmission scenario defined in (2), the destination applies the conventional matched filter [11] as $h_{s, d}^{*} y_{s, d}$. The output SNR, denoted as $\gamma$, can be computed as

$$
\gamma^{D}=\frac{P}{N_{0}+\alpha P}\left|h_{s, d}\right|^{2} .
$$

In the perfect channel estimation scenario, i.e., $\alpha=0$, the SNR at the destination increases with the data transmission power $P$. However with channel estimation error, increasing the data transmission power cannot lead to arbitrarily large SNR. This limits the performance of the direct transmission scenario and causes dramatic performance degradation. We 
also note that the effect of the channel estimation error, which is $\alpha P$ in (5), increases with high data transmission power. Motivated by the bad impact of channel estimation error on the direct transmission scenario, we investigate in the next section the ability of the cooperative transmission scenario to mitigate such impact.

\section{EFFect of Cooperative Communications}

In this section, we analyze the performance of the direct and cooperative transmission scenarios introduced in Section II. For each scenario, we calculate the outage probability and the SNR gap ratio, which is defined as

$$
R=\frac{\left.\gamma\right|_{(\alpha=0)}-\left.\gamma\right|_{(\alpha \neq 0)}}{E\left\{\left.\gamma\right|_{(\alpha=0)}\right\}}
$$

where $E\{$.$\} denotes the statistical expectation of a particular$ random variable. Intuitively, the SNR gap ratio measures the reduction in the $\operatorname{SNR},\left(\left.\gamma\right|_{(\alpha=0)}-\left.\gamma\right|_{(\alpha \neq 0)}\right)$, compared to the average SNR without channel estimation error, i.e., it measures the relative SNR gap ratio.

For the direct transmission scenario defined in (2), the output SNR in (5) is an exponential random variable with mean $\left(P \delta_{s, d}^{2}\right) /\left(N_{0}+\alpha P\right)$, i.e., $\gamma^{D} \sim \exp \left(\left(N_{0}+\alpha P\right) /\left(P \delta_{s, d}^{2}\right)\right)$. The outage probability, which is defined as the probability that the output SNR is less than a particular threshold $\gamma_{t h}$, is computed as

$$
F_{\gamma^{D}}\left(\gamma_{t h}\right)=\operatorname{Pr}\left(\gamma^{D} \leq \gamma_{t h}\right)=1-\exp \left(-\frac{N_{0}+\alpha P}{P \delta_{s, d}^{2}} \gamma_{t h}\right)
$$

By substituting (5) into (6), the direct transmission SNR gap ratio can be written as

$$
R^{D}=\frac{\alpha P}{\delta_{s, d}^{2}\left(N_{0}+\alpha P\right)}\left|h_{s, d}\right|^{2} .
$$

The source-destination channel gain squared $\left|h_{s, d}\right|^{2}$ is an exponential random variable with mean $\delta_{s, d}^{2}$. Hence, the direct transmission SNR gap ratio in (8) is an exponential random variable, i.e., $R^{D} \sim \exp \left(\left(N_{0}+\alpha P\right) /(\alpha P)\right)$. Finally, the average SNR gap ratio can be calculated as

$$
E\left\{R^{D}\right\}=\frac{\alpha P}{N_{0}+\alpha P} .
$$

In the cooperative transmission scenario, the destination applies maximal-ratio combining (MRC) [12] to coherently combine the signals received from the source and the $N$ relays. The output of the MRC detector at the destination is given by

$$
y^{C}=\frac{\sqrt{P_{0}}}{N_{0}+\alpha P_{0}} h_{s, d}^{*} y_{s, d}^{C}+\sum_{i=1}^{N} \frac{\sqrt{\tilde{P}_{i}}}{N_{0}+\alpha \tilde{P}_{i}} h_{r_{i}, d}^{*} y_{r_{i}, d}^{C} .
$$

Let $\tilde{\mathbf{P}}=\left[P_{0}, \tilde{P}_{1}, \cdots, \tilde{P}_{N}\right]^{T}$ denote the power distribution vector, where $T$ denotes vector transpose. For a fixed power vector $\tilde{\mathbf{P}}$, the conditional SNR can be computed as

$$
\gamma^{C}(\tilde{\mathbf{P}})=\frac{P_{0}}{N_{0}+\alpha P_{0}}\left|h_{s, d}\right|^{2}+\sum_{i=1}^{N} \frac{\tilde{P}_{i}}{N_{0}+\alpha \tilde{P}_{i}}\left|h_{r_{i}, d}\right|^{2} .
$$

In the sequel, we obtain the distribution of the power vector $\tilde{\mathbf{P}}$, which is based on the transmission between the user and the $i$-th relay, modeled in (3). Without loss of generality, we assume M-PSK modulation type. The conditional SER at the $i$-th relay, which is conditioned on the the channel coefficient $h_{s, r_{i}}$, can be written as [13]

$$
\varepsilon^{h_{s, r_{i}}}=\Psi\left(\gamma_{i}\right)=\frac{1}{\pi} \int_{0}^{(M-1) \pi / M} \exp \left(-\frac{b \gamma_{i}}{\sin ^{2} \theta}\right) d \theta,
$$

where $\gamma_{i}=P_{0}\left|h_{s, r_{i}}\right|^{2} /\left(N_{0}+\alpha P_{0}\right)$ is the instantaneous SNR at the $i$-th relay and $b=\sin ^{2}(\pi / M)$. By averaging (12) with respect to the exponential random variable $\left|h_{s, r_{i}}\right|^{2}$, the SER can be given by

$$
\varepsilon=F_{1}\left(1+\frac{b P_{0} \delta_{s, r_{i}}^{2}}{\left(N_{0}+\alpha P_{0}\right) \sin ^{2} \theta}\right),
$$

where $F_{1}(x(\theta))=1 / \pi \int_{0}^{(M-1) \pi / M} 1 / x(\theta) d \theta$.

As described in Section II, the $i$-th relay retransmits the source's symbol only if it has correctly decoded that symbol. Hence the power of the $i$-th relay, $\tilde{P}_{i}, i=1,2 \cdots, N$, is distributed as a Bernoulli random variable with success probability equal to $(1-\varepsilon)$, i.e.,

$$
\tilde{P}_{i}=\left\{\begin{array}{lll}
P_{i} & \text { w.p. } & 1-F_{1}\left(1+\frac{b P_{0} \delta_{s, r_{i}}^{2}}{\left(N_{0}+\alpha P_{0}\right) \sin ^{2} \theta}\right) \\
0 & \text { w.p. } \quad F_{1}\left(1+\frac{b P_{0} \delta_{s, r_{i}}^{2}}{\left(N_{0}+\alpha P_{0}\right) \sin ^{2} \theta}\right)
\end{array},\right.
$$

where w.p. stands for "with probability". We note that the relays' powers $\tilde{P}_{i}, i=1,2 \cdots, N$ are independent random variables since each one depends on its own source-relay channel gain $\left|h_{s, r_{i}}\right|$, which are independent of each other as assumed in Section II.

Let $h_{0}=\left|h_{s, d}\right|^{2} / \delta_{s, d}^{2}$ and $h_{i}=\left|h_{r_{i}, d}\right|^{2} / \delta_{r_{i}, d}^{2}, i=$ $1,2, \cdots, N$, where $h_{i}, i=0,1, \cdots, N$, is distributed as a zero-mean complex Gaussian random variable with unit variance. By averaging the conditional SNR in (11) with respect to $\tilde{\mathbf{P}}$, the cooperative transmission SNR can be obtained as

$$
\gamma^{C}=\sum_{i=0}^{N} a_{i}\left|h_{i}\right|^{2}
$$

where $a_{0}=\frac{P_{0} \delta_{s, d}^{2}}{N_{0}+\alpha P_{0}}$ and $a_{i}=\frac{P_{i} \delta_{r_{i}, d}^{2}}{N_{0}+\alpha P_{i}}\left(1-F_{1}(1+\right.$ $\left.\left.\frac{b P_{0} \delta_{s, r_{i}}^{2}}{\left(N_{0}+\alpha P_{0}\right) \sin ^{2} \theta}\right)\right), i=1,2, \cdots, N$. Furthermore, the outage probability is calculated as

$$
F_{\gamma^{C}}\left(\gamma_{t h}\right)=\sum_{i=0}^{N} b_{i}\left(1-\exp \left(-\frac{\gamma_{t h}}{a_{i}}\right)\right)
$$

where $b_{i}=\prod_{k=0, k \neq i}^{N} \frac{a_{i}}{a_{i}-a_{k}}, i=0,1, \cdots, N$.

By substituting (15) into (6), the cooperative transmission SNR gap ratio can be given by

$$
R^{C}=\frac{\left.\gamma^{C}\right|_{(\alpha=0)}-\gamma^{C}}{E\left\{\left.\gamma^{C}\right|_{(\alpha=0)}\right\}}=\sum_{i=0}^{N} c_{i}\left|h_{i}\right|^{2},
$$




\begin{tabular}{|l|c|}
\hline Parameter & Value \\
\hline \hline Cell radius & $1 \mathrm{~km}$ \\
\hline Thermal noise & $-100 \mathrm{dBm}$ \\
\hline Max transmission power & $25 \mathrm{dBm}$ \\
\hline Propagation model & $31.5+35 \log _{10}(\mathrm{~d}$ in $\mathrm{m}) \mathrm{dB}$ \\
\hline
\end{tabular}

TABLE I

Simulation parameters of a typical cellular system.

where

$$
\begin{aligned}
c_{0} & =\frac{\delta_{s, d}^{2}}{S} \frac{\alpha P_{0}^{2}}{N_{0}\left(N_{0}+\alpha P_{0}\right)}, \\
c_{i} & =\frac{\delta_{r_{i}, d}^{2}}{S}\left(\frac{\alpha P_{i}^{2}}{N_{0}\left(N_{0}+\alpha P_{i}\right)}-\frac{P_{i}}{N_{0}} F_{1}\left(1+\frac{b P_{0} \delta_{s, r_{i}}^{2}}{N_{0} \sin ^{2} \theta}\right)\right. \\
& \left.+\frac{P_{i}}{N_{0}+\alpha P_{i}} F_{1}\left(1+\frac{b P_{0} \delta_{s, r_{i}}^{2}}{\left(N_{0}+\alpha P_{0}\right) \sin ^{2} \theta}\right)\right),
\end{aligned}
$$

in which $S=E\left\{\left.\gamma^{C}\right|_{(\alpha=0)}\right\}=\left.\sum_{i=0}^{N} a_{i}\right|_{(\alpha=0)}$. The cooperative transmission SNR gap ratio defined in (17) represents a weighted sum of a set of independent chi-square random variables [11] and its probability density function (PDF) can be written as

$$
f_{R^{C}}(r)=\sum_{i=0}^{N} \frac{d_{i}}{c_{i}} \exp \left(-\frac{r}{c_{i}}\right) U(r),
$$

where $d_{i}=\prod_{k=0, k \neq i}^{N} \frac{c_{i}}{c_{i}-c_{k}}, i=0,1, \cdots, N$. Finally, the average of the cooperative transmission SNR gap ratio is computed as

$$
E\left\{R^{C}\right\}=\sum_{i=0}^{N} c_{i}
$$

\section{NUMERICAL RESUlts}

In this section, we present some numerical results to illustrate the impact of the cooperative transmission scheme on the channel estimation error. The outage probability and SNR gap ratio are utilized to characterize such impact. For fair comparison, we assume that a total power $P$ is available for the direct and cooperative transmission scenarios. We assume maximum of $N=6$ relays are available and we consider power allocation policy, in which $P_{0}=P / 2$ and $P_{i}=P /(2 N), i=1,2, \cdots, N$ [4]. QPSK modulation type is assumed throughout this paper. Table I summarizes a typical set of simulation parameters for cellular networks. Finally, the shown results are averaged over 1000 independent network realizations, where the locations of the users and the relays are randomly distributed in each realization.

We assume that the channel estimation error variance is $\alpha=0.05$. As indicated in Section II, the channel estimation error variance, $\alpha$, is fixed and does not depend on the data transmission power, $P$. Fig. 2 depicts the outage probability, given by (7) and (16) for the direct and cooperative transmission scenarios, respectively, at $P / N_{0}=20 \mathrm{~dB}$. As shown, the direct transmission has the highest outage probability for any SNR threshold, $\gamma_{t h}$. It is also shown that as the number of relays increases, the cooperative transmission outage probability

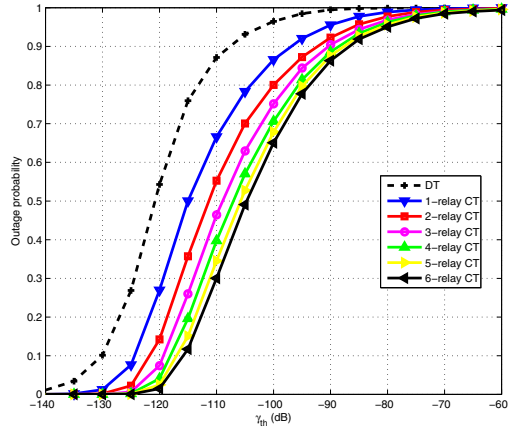

Fig. 2. Outage probability of the direct and cooperative transmission scenarios for $\alpha=0.05$ and $P / N_{0}=20 \mathrm{~dB}$. Cooperative transmission reduces the outage probability as the number of relays increases.

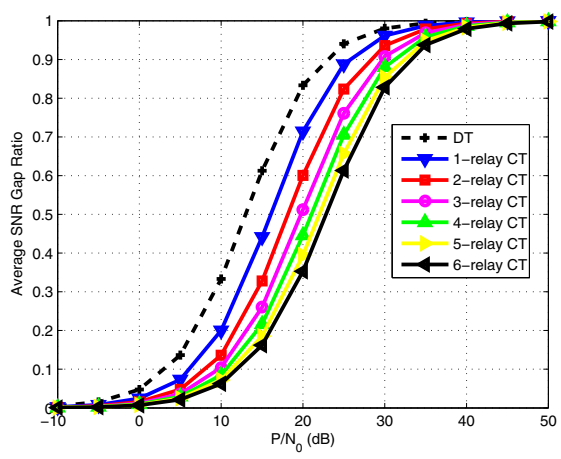

Fig. 3. Average SNR gap ratio of the direct and cooperative transmission scenarios for $\alpha=0.05$. Cooperative transmission reduces the average SNR gap ratio as the number of relays increases.

reduces. This is due to the fact that cooperative transmission with $N$ relays provides $N+1$ independently-faded paths from the source to the destination. Hence, diversity order $N+1$ is achieved.

In addition to the outage probability, the average SNR gap ratio is of great interest. Fig. 3 depicts the average SNR gap ratio for the different transmission scenarios as a function of $P / N_{0}$. For fixed $N_{0}$, Fig. 3 shows that the average SNR gap ratio increases with the data transmission power, $P$. This is due to the fact that the channel estimation error effect, which is $\alpha P$ in (2), is more significant at high transmission power compared to the noise variance. At high transmission power, the average SNR gap ratio is 1 as can be shown using (9). It is also depicted in Fig. 3 that the direct transmission scenario has the largest SNR gap ratio compared to the cooperative transmission scenario. Furthermore, increasing the number of utilized relays reduces the average SNR gap ratio. At $P / N_{0}=10 \mathrm{~dB}$, the direct transmission scenario suffers SNR gap ratio of 0.33 , while the cooperative transmission scenario with $N=6$ relays suffers SNR gap ratio of 0.06 . From Fig. 3, we conclude that the cooperative communication protocol reduces the effect of the channel estimation error, which is one of the main results of this paper. 


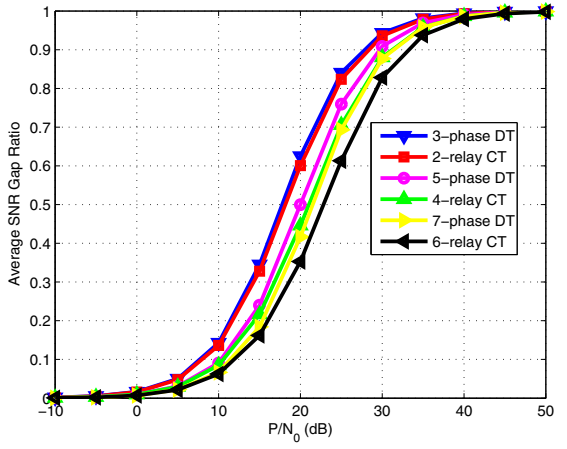

Fig. 4. Average SNR gap ratio of the multi-phase direct and cooperative transmission scenarios for $\alpha=0.05$. Cooperative transmission reduces the SNR gap ratio more than the multi-phase direct transmission for the same number of phases.

\section{A. Multi-phase Direct transmission}

As a way to explain why cooperative communications reduce the effect of channel estimation error, we consider in this subsection the multi-phase direct transmission scenario. In this scheme, a user sends its data to its destination in $N$ consecutive channel uses, each with a transmission power of $P / N$. There is no relays utilized in this scheme. Similar to (8), it can be shown that the SNR gap ratio is given by

$$
R^{D}(N)=\frac{\alpha P}{N \delta_{s, d}^{2}\left(N_{0}+\alpha P / N\right)}\left|h_{s, d}\right|^{2},
$$

i.e., $R^{D}(N) \sim \exp \left(N\left(N_{0}+\alpha P / N\right) /(\alpha P)\right)$. The average SNR gap ratio can be calculated as

$$
E\left\{R^{D}(N)\right\}=\frac{\alpha P}{N\left(N_{0}+\alpha P / N\right)} .
$$

We note that the multi-phase direct transmission scenario achieves diversity order equal to 1 , and has outage probability similar to that of the direct transmission. Fig. 4 depicts the average SNR gap ratio for the multi-phase and cooperative transmission scenarios. As shown, the multi-phase direct transmission protocol reduces the SNR gap ratio as the number of phases increases. Therefore by distributing the total transmission power across multiple transmission phases, the effect of the channel estimation error can be mitigated. In Fig. 4, it is also shown that the cooperative transmission scenario reduces the SNR gap ratio more compared to the multi-phase direct transmission, for the same total number of transmission phases.

From Fig. 3 and Fig. 4, we conclude that the reduction in the SNR gap ratio is due to two main factors. The first factor is the distribution of the transmission power across multiple transmission phases. This reduces the transmission power in each phase, and accordingly the channel estimation error portion, $\alpha P$, in each transmission is reduced. This first reduction factor exists in both the multi-phase direct transmission and cooperative transmission scenarios, and hence both of them mitigate the effect of channel estimation error by increasing the number of transmission phases as was shown in Fig. 4.

The second factor of reducing the effect of channel estimation error is the achieved diversity order. The cooperative transmission scenario utilizes relays, other than retransmission over the same channel. The cooperation gain resulting from utilizing relays reduces the channel estimation error effect more. Since the cooperative scheme achieves full diversity order along with distributing the transmission power, it reduces the SNR gap ratio compared to that of the multi-phase direct transmission, as was shown in Fig. 4.

\section{CONCLUSION}

In this paper, we have investigated the impact of the cooperative communications on mitigating channel estimation error effect. The SNR gap ratio, which measures the reduction in the SNR, and the outage probability were utilized to characterize the system performance. We have shown that the cooperative transmission is less susceptible to the channel estimation error compared to the direct transmission. Furthermore, increasing the number of relays results in lower SNR gap ratio. At $P / N_{0}=10 \mathrm{~dB}$ and channel estimation error variance $\alpha=$ 0.05 , the direct transmission scenario suffers SNR gap ratio of 0.33 , while the cooperative transmission scenario with $N=6$ relays suffers SNR gap ratio of 0.06 only. Finally, we have illustrated that cooperative transmission reduces the channel estimation error effect due to two main factors: (1) achieving spatial diversity via relays and (2) distributing the transmission power across multiple transmission phases.

\section{REFERENCES}

[1] K. J. R. Liu, A. K. Sadek, W. Su, and A. Kwasinski, Cooperative communications and networking, Cambridge University Press, 2008.

[2] J. N. Laneman, D. N. C. Tse, and G. W. Wornell, "Cooperative diversity in wireless networks: efficient protocols and outage behavior," IEEE Trans. Information Theory, vol. 50, no. 12, pp. 3062-3080, Dec. 2004.

[3] W. Su, A. K. Sadek, and K. J. R. Liu, "Cooperative communication protocols in wireless networks: performance analysis and optimum power allocation," Wireless Personal Communications, vol. 44, pp. 181217, Jan. 2008.

[4] A. K. Sadek, W. Su, and K. J. R. Liu, "Multi-node cooperative communications in wireless networks," IEEE Trans. Signal Processing, vol. 55, pp. 341-355, Jan. 2007.

[5] A. S. Ibrahim, A. K. Sadek, W. Su, and K. J. R. Liu, "Cooperative communications with partial channel state information: when to cooperate?," Proc. IEEE Global Telecommunications Conference (Globecom'05), vol. 5, pp. 3068 - 3072, Nov. 2005.

[6] A. S. Ibrahim, A. K. Sadek, W. Su, and K. J. R. Liu, "Cooperative communications with relay-selection: when to cooperate and whom to cooperate with?," IEEE Trans. on Wireless Comm., vol. 7, pp. 2814 2827, Jul. 2008.

[7] J. N. Laneman and G. W. Wornell, "Distributed space-time coded protocols for exploiting cooperative diversity in wireless networks," IEEE Trans. Information Theory, vol. 49, no. 10, pp. 2415-2425, Oct. 2003.

[8] K. G. Seddik, A. K. Sadek, A. S. Ibrahim, and K. J. R. Liu, "Design criteria and performance analysis for distributed space-time coding," IEEE Trans. on Vehicular Technology, vol. 57, pp. 2280 - 2292, Jul. 2008.

[9] H. Cheon and D. Hong, "Effect of channel estimation error in OFDMbased WLAN," IEEE Communications Letters, vol. 6, pp. 190 - 192, May 2002.

[10] H. Jin, R. Laroia, and T. Richardson, "Superposition by position," Proc. IEEE Information Theory Workshop, pp. 222 - 226, Mar. 2006.

[11] J. G. Proakis, Digital Communications, McGraw-Hill Inc., 4th ed., 2000.

[12] D. G. Brennan, "Linear diversity combining techniques," Proc. IEEE, vol. 91, no. 2, pp. 331-356, Feb. 2003.

[13] M.K. Simon and M.-S. Alouini, "A unified approach to the performance analysis of digital communication over generalized fading channels," Proc. IEEE, vol. 86, pp. 1860-1877, Sep. 1998. 